\title{
Beyond Serrated Flow in Bulk Metallic Glasses: What Comes Next?
}

\begin{abstract}
R. MAAB
This manuscript is based on an oral contribution to the TMS 2020 annual meeting and is dedicated to Prof. Peter Liaw, who for decades has shown great interest in serrated plastic flow. Here we will focus on the case of bulk metallic glasses, and begin with briefly summarizing some aspects of serrated and non-serrated inhomogeneous flow-a phenomenon that has perplexed materials scientists for decades. Four directions of research are identified that emerged out of the desire to fundamentally understand the intermittent inhomogeneous flow response. These research directions gear away from the phenomenological stress-strain behavior but put the underlying shear defect into focus. Unsolved problems and future research topics are discussed.
\end{abstract}

https://doi.org/10.1007/s11661-020-05985-w

(C) The Author(s) 2020

\section{INTRODUCTION}

IN the early two thousands, the topic of serrated versus non-serrated flow in bulk metallic glasses (BMGs) experienced a second wave of interest following the remarkable initial work by Kimura and Masumoto presented more than 20 years earlier. ${ }^{[1-3]}$ Kimura and Masumoto recognized that the appearance of plastic flow in BMGs at low homologous temperatures (the inhomogeneous deformation regime) can be either smooth or intermittent, depending on the applied deformation rate and/or deformation temperature. In essence, this is phenomenologically very similar to dynamic strain aging of, for example, steels ${ }^{[4]}$ or Al-Mg alloys ${ }^{[5]}$ where the interplay between depinning of dislocations and the re-trapping of them by solutes gives rise to the so-called jerky flow curves for particular combinations of the deformation rate and testing temperature. In fact, our recent efforts suggest that intermittent single-crystal microplasticity is another manifestation of a rather generic coupling between the far-field rate and some underlying deformation kinetics. ${ }^{[6]}$ In this latter case, crystallographic slip is governed by the collective dislocation velocity during the dynamic (also referred to as an avalanche) deformation phase.

R. MAAB is with the Department of Materials Science and Engineering and Frederick Seitz Materials Research Laboratory, University of Illinois at Urbana-Champaign, Urbana, IL 61801, and also with the Federal Institute of Materials Research and Testing (BAM), Unter den Eichen 87, 12205 Berlin, Germany. Contact e-mail: rmaass@illinois.edu; robert.maass@bam.de

Manuscript submitted May 29, 2020.

Article published online September 8, 2020
Whilst the macroscopic emergence of the stress-strain response has some similarities in all these cases (metallic glasses, dynamic strain aging, single crystal microplasticity), the underlying physics is clearly different.

Kimura and Masumoto established via tearing, bending, and compression testing of BMGs across different temperatures that two regimes appear in an extension rate vs. 1/T representation. At constant applied deformation rate, serrated flow was observed at higher temperatures, and at lower temperatures non-serrated flow occurred. The border between the regimes decreased in temperature with decreasing applied rate, and a simple Arrhenius relationship captured this trend, with effective barrier energies that were alloy specific and in the range of 0.3 to $0.5 \mathrm{eV}$. These efforts did not only reveal the thermally-activated nature of inhomogeneous flow of metallic glasses, but also included a detailed mechanical understanding on how both sample and mechanical compliances affect the serration amplitude. ${ }^{[3]}$

About 25 years later and driven by the desire to fundamentally understand the (micro)-structural mechanisms during strain localization (shear banding) of metallic glasses, the transition from serrated to non-serrated flow received renewed attention. With the vision of finding structural parameters or properties that potentially could improve the plastic strain prior to failure, fundamental work focused on questions related to shear-band nucleation dynamics, ${ }^{[7-9]}$ how shear advances (front propagation or shear-displacement jump),${ }^{[10]}$ the duration of shear-band propagation, ${ }^{[11-14]}$ or heat generation and dissipation during shear-band propagation. ${ }^{[15,16]}$ Gaining deeper and fundamental insight into these topics imposed significant 
experimental challenges, due to the nanoscopic damage localization in a disordered material and the short time-scales of shear banding.

An important contribution to both the speed at which shear bands operate and the corresponding heating from plastic work was made by Wright et al. ${ }^{[1]}$ Using strain gauges directly mounted on the sample, it was convincingly demonstrated that the duration of a shear event is of the order of a milli-second, resulting in shear-band velocities orders of magnitude smaller than the shearwave speed $(\sim \mathrm{km} / \mathrm{s})$. This insight lead to significant corrections of earlier proposed shear-band heating models that relied on shear durations in the range of $10^{-10}$ to $10^{-6}$ seconds, ${ }^{[17-19]}$ and which resulted in local temperature excursions up to the glass transition temperature $\left(T_{g}\right)$ or even exceeding several thousand Kelvin.

With shear durations in the milli-second regime, it became possible to directly trace the spatiotemporal profile of shear-band dynamics when using measurement systems with sufficiently short electronic and mechanical response times. We pursued this route and began to trace the time-resolved shear-band dynamics across temperature and various metallic glass alloys. $^{[20-22]}$ One of the central outcomes of this effort was that the logarithm of the average shear-band velocity, as derived from a simple linearization of the underlying displacement jump and by adopting a shear-displacement jump mechanism, scaled linearly when graphed vs. 1/T. Clearly, this represented nothing else than the two decades earlier mapped transition between serrated and non-serrated flow by Kimura and Masumoto. However, now their "deformation map" and the derived effective barrier energy, $E_{\text {eff }}$, was directly linked to the propagation speed (sliding) of the shear defect itself. Furthermore, the transition from serrated to non-serrated flow could be explained as a result of competing velocities: as long as the shear-band velocity $\left(v_{\mathrm{SB}}\right)$ remained larger than the resolved cross-head velocity $\left(v_{\mathrm{XH}}\right)$ of the testing machine, serrated flow occurred. When both velocities become equal, the transition to non-serrated inhomogeneous flow begins. Beyond this point, the $v_{\mathrm{SB}}$ is slower than $v_{\mathrm{XH}}$ and the measured shear velocity will always equate to the resolved cross-head velocity. Thus, the transition temperature, $T_{\text {crit }}$, is sensitive to $v_{\mathrm{XH}}$ for one and the same alloy. Figure 1 summarizes this finding schematically.

From the perspective of the shear-band material, the result embodied in Figure 1 can be understood in terms of a competition between a material- and temperature-dependent relaxation rate and a (athermal) disordering rate imparted by the shear deformation. Since these rates are shown to sensitively depend on small variations of the external temperature, internal temperature excursions and thus shear-band heating must indeed be small. State-of-the-art thermal imaging supports this conclusion. ${ }^{[23,24]}$ The thermal-activation framework of serrated flow can be expanded when considering a less recognized insight gained from our shear-band dynamics work, namely that shear-band aging (structural relaxation) proceeds during the elastic

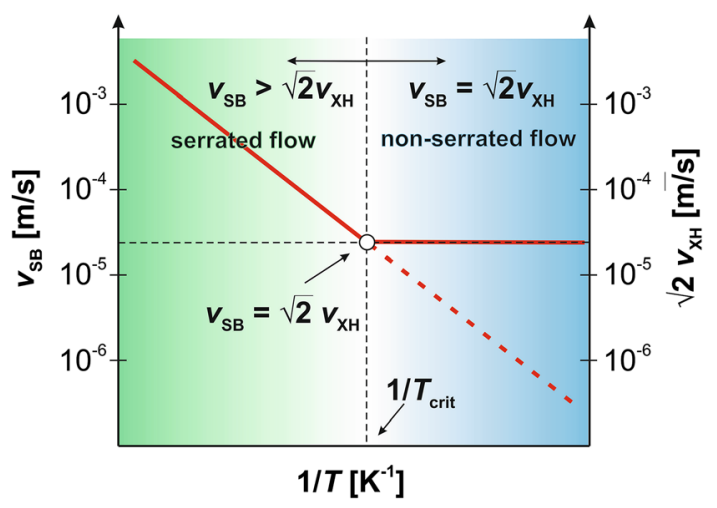

Fig. 1-Schematic indicating how the deformation mode of a metallic glass transitions from inhomogeneous serrated to inhomogeneous non-serrated flow at an alloy-specific critical temperature below which the shear-band velocity is equal or below the applied deformation rate. Deformation is thus driven in the non-serrated regime (Color figure online).

loading segments $\left(v_{\mathrm{SB}} \ll v_{\mathrm{XH}}\right)$ in-between individual serrations. ${ }^{[25]}$ Similar to the shear-band propagation phenomenon, thermal activation with its distinct energy scale can be shown to govern shear-band aging. Structural relaxation during the nominally elastic segments between stress drops give thus the shear-band material the ability to recover. With the absence of any other internal damage mechanisms, this shear-band aging response should allow a number of serration-cycles that mainly (ignoring moments due to lateral shear) is limited by the physical dimension of the specimen. Final fracture after a small number of serrations must therefore be promoted by other damage processes, such as internal micro-cracking, ${ }^{[26,27]}$ than shear itself. Another consequence of an increasing $v_{\mathrm{XH}}$ is therefore that the aging time during reloading shortens, thereby reducing the serration stress-amplitude towards $T_{\text {crit }}$ (negative strain-rate sensitivity). This sequence of sliding $\left(v_{\mathrm{SB}} \gg v_{\mathrm{XH}}\right)$ followed by arrest $\left(v_{\mathrm{SB}} \ll v_{\mathrm{XH}}\right)$ can be well described with the characteristics of a stick-slip system, where mechanical energy storage is followed by a release of elastic energy. As evident from the machine equation used to describe stick-slip systems, the slip phase is depending on the compliance, which in the case of the mechanical testing experiment includes both the sample and the machine. This needs to be kept in mid, as the property $v_{\mathrm{SB}}$ is consequently not intrinsic to the material. However, our understanding is that the Arrhenius construct offers a pathway to assess an intrinsic property of the slip phase (shear-band propagation), namely $E_{\mathrm{eff}}$. In view of the field's central vision to design metallic glasses with large intrinsic ductility, this intrinsic $E_{\text {eff }}$ becomes an interesting quantity as it seems to govern both shear-resistance and shear-band recovery. This will be discussed in more detail later.

The afore summarized findings should be viewed as a brief introduction to the central question of this article: What research questions come beyond serrated flow in metallic glasses? Based on a contribution to a 
symposium in honor of Prof. Peter Liaw at the TMS 2020 annual meeting, I discuss this question non-exhaustively by identifying the following four main research areas:

A. the statistical signature of serrated flow,

B. the value of $E_{\text {eff }}$ as a structural parameter that possibly quantifies ductility,

C. the structural length scale, and

D. the structure of the shear defect underlying inhomogeneous deformation.

Without doubt, continued efforts in these areas will greatly enhance our understanding of inhomogeneous plasticity in metallic glasses and will lead us closer to the visionary goal of designing ductile metallic glasses.

I write this article as a viewpoint and in tribute to Prof. Liaw's leadership in the community and his never ceasing interest in serrated flow.

\section{BEYOND SERRATED FLOW IN BULK METALLIC GLASSES}

Nine years have now passed since the finding schematically summarized on Figure 1, and five since we summarized the full body of shear-band dynamics work. ${ }^{[22]}$ As of today, the body of literature on serrated flow continues to grow for metallic glasses, and also finds renewed interested in the novel area of multi-principle element alloys. ${ }^{[28-30]}$ In contrast, our own continued efforts departed from the stress-strain signature of serrated flow, targeting fundamentals of strain localization in metallic glasses, some of which are contained in the following subsections that are organized according to the identified research areas A to D.

\section{A. Statistical Signature of Serrated Flow}

The generic feature of plastic discontinuities in a stress-strain response of structural materials has recently attracted the statistical physics community ${ }^{[31]}$ and was subject of our review on microplasticity. ${ }^{[32]}$ Central to these works is the attempt to describe fluctuations in plasticity (or simply the discrete displacement increment, often referred to as a (slip)-avalanche) via analytical models that rely on little or no microstructural details. Such models propose power-law scaling of the form $D(S) \sim S^{-\alpha}$, with $S$ being the event size and where a numerically identical and trivial scaling exponent $\alpha$ can give the attribute "universal" to such distribution functions $D(S)$. Prior to successfully applying this approach to metallic glasses, other intermittently deforming materials had been demonstrated to follow scale-free like universal statistics, including for example single crystal plasticity ${ }^{[33]}$ and slip of granular media. ${ }^{[3]}$ These developments are somewhat at odds with our classical approach to plasticity that implicitly relies on well-defined means and scales. Indeed, truncated power-law scaling, and therefore scale-free (like) behavior was found for several BMG-compositions. ${ }^{[35-37]}$ Other compositions seem to yield serrated flow statistics with scale-dependent distributions. ${ }^{[38]}$ Noteworthy is the finding that experimental tuning parameters, such as strain rate, determine the size of the largest observed shear events, ${ }^{[39]}$ which in fact is very much in agreement with the earlier discussed shear-band aging and related reduction in stress-drop magnitude with increasing rate. Consequently, similar trends are expected upon decreasing the temperature.

The discrepancy between different reports as to whether a monolithic metallic glass exhibits scale-free intermittent flow and therefore can be framed into the context of critical phenomenon remains a puzzling topic. This can nicely be demonstrated by the work of Sun et al., ${ }^{[35]}$ who tested a series of compositions,

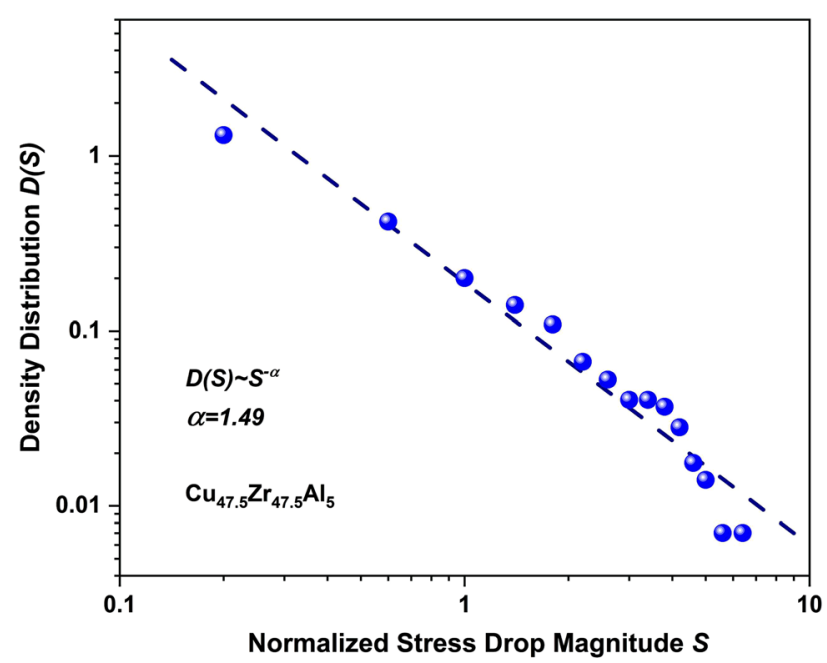

(a)

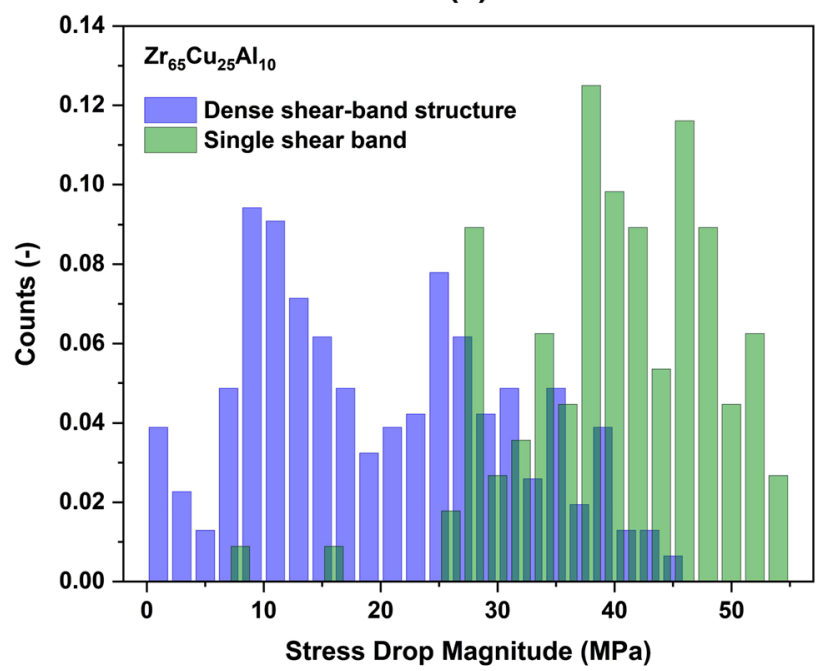

(b)

Fig. 2 - (a) Density distribution $D(S)$ vs. normalized stress-drop magnitude $S$ for $\mathrm{Cu}_{47.5} \mathrm{Zr}_{47,5} \mathrm{Al}_{5}$ revealing power-law scaling. Data kindly provided by B.A. Sun. Reproduced with permission from Reference 35 Copyright (2010) by the American Physical Society. (b) normalized histograms for stress-drop magnitudes from compressively deformed $\mathrm{Zr}_{65} \mathrm{Cu}_{25} \mathrm{Al}_{10}$ with a dense shear-band structure (blue) and a single system-spanning shear-band (green). Reproduced from Reference 38 with permission from Elsevier (Color figure online). 
including the same alloy in different annealing states. Figure 2(a) displays data from Reference 35 that evidences power-law scaling of a density distribution with an exponent of approx. 1.5 for $\mathrm{Cu}_{47.5} \mathrm{Zr}_{47.5} \mathrm{Al}_{5}$. A similar exponent was found for a subset of the tested alloys, all of which exhibited the largest strains at failure $(>10 \mathrm{pct})$ during compressive loading.

Yet another subset of alloys yielded stress-drop distributions that were referred to as chaotic, which simply indicates no scale-free signature even though the functional form of the distribution was not specifically evaluated. Interestingly, an alloy from this group was subjected to mechanical pre-loading, after which the stress-drop magnitude-distribution became scale-free. In agreement with the other alloys that revealed power-law distribution, a large plastic strain was also reported. This difference in statistical signature is linked to the number of shear bands and their interaction during plastic flow. Only alloys with a dense-shear band structure are admitting sufficiently large numbers of small stress-drops (serrations) that can promote scalefree scaling. Since aspect ratio and sample alignment significantly determine strain at failure, ${ }^{[40,41]}$ one could conclude that the manifestation of different serration statistics may merely be results of boundary conditions that cause inhomogeneous stress states. It is noted that the above mentioned mechanically pre-loaded alloy is such a case.

However, the situation is not so clear. Our own work on $\mathrm{Zr}_{65} \mathrm{Cu}_{25} \mathrm{Al}_{10}$ revealed that significant changes in boundary conditions (from uncontrolled constrained to unconstrained) only shifted the serration-magnitude histogram (Figure 2(b)). At the same time, the plastic strains were in both cases largely exceeding 10 pct. Furthermore, a variation of aspect ratio between 2:1 and 1:2 (height:diameter) did not show any power-law scaling. ${ }^{[42]}$ Since an aspect-ratio reduction of a malleable BMG constitutes the extreme case of shear-band interaction due to an insufficient distance between free surfaces for unconstrained system-spanning shear-band formation, one may have expected a power-law scaling if the introduction of inhomogeneous stress fields is the sole factor determining the serration statistics. This does, however, not seem to be the case. There is thus a structural component in monolithic BMGs that contributes to the scaling as shown in Figure 2(a) that so far remains fully undefined. This is much different to the case of metallic-glass matrix composites, where the statistics of stress-strain instabilities changes towards smaller event sizes ${ }^{[43,44]}$ _ a result that naturally emerges due to the introduction of internal micro-structural length scales where crystallites lead to shear-band deflection and arrest. ${ }^{[45]}$ In fact, some first evidence has been provided that even the scaling exponent $\alpha$ directly scales with the volume fraction and average size of the crystalline phase. ${ }^{[45]}$ Such clear findings are lacking in the case of monolithic metallic glasses. Instead, the small amount of published work, the challenge of properly determining the distribution forms, and the unassessed effects of testing parameters, render the emergence of power-law (like) scaling of serrated flow in the monolithic case at most phenomenologically understood. In addition, an inherent conflict surfaces when considering that one essential message from the agreement between scale-free statistical models with universal exponents is the insensitivity to microstructural details, whereas the above discussion suggests at least some structural dependencies for monolithic glasses. Furthermore, scale-invariance of plasticity entails some underlying mechanism with long-range coupling and correlated structural activity. Why this may be the case in some monolithic glassy alloys, whereas in others not, remains unsolved. This unsatisfactory situation reminds us strongly about crystalline microplasticity $^{[32]}$ that initially found strong agreement with universal scaling-laws across numerous microstructure, whereas we first now begin to understand the opportunities hidden in the microstructurally sensitive statistical scaling laws of plastic fluctuations. ${ }^{[46,47]}$ With enough careful continued research efforts, the same may eventually be concluded for metallic glasses, which interestingly would imply the presence of unexpectedly large structure-dependent internal length scales in monolithic metallic glasses.

\section{B. Effective Barrier Energies Across Numerous Alloy Systems}

The slope of the data in Figure 1 determines an effective barrier energy $E_{\text {eff }}$ that can be seen as a measure of the resistance to shear. This is better understood when acknowledging that data sets for numerous different alloys seem to converge at $1 / T=0$. Extrapolating to this point at high temperatures, the shear-band velocity attains a value of $\mathrm{km} / \mathrm{s}$ that is not much different than the sound velocity. Given a converging intersection point on the ordinate, a larger value of $E_{\mathrm{eff}}$ means that shear is much slower at a fixed temperature than for a smaller $E_{\text {eff }}$. Figure 3 demonstrates this for the $\mathrm{Zr}_{\mathrm{x}} \mathrm{Cu}_{90-\mathrm{x}} \mathrm{Al}_{10}$ system, where approximately an order of magnitude difference in shear-velocity is

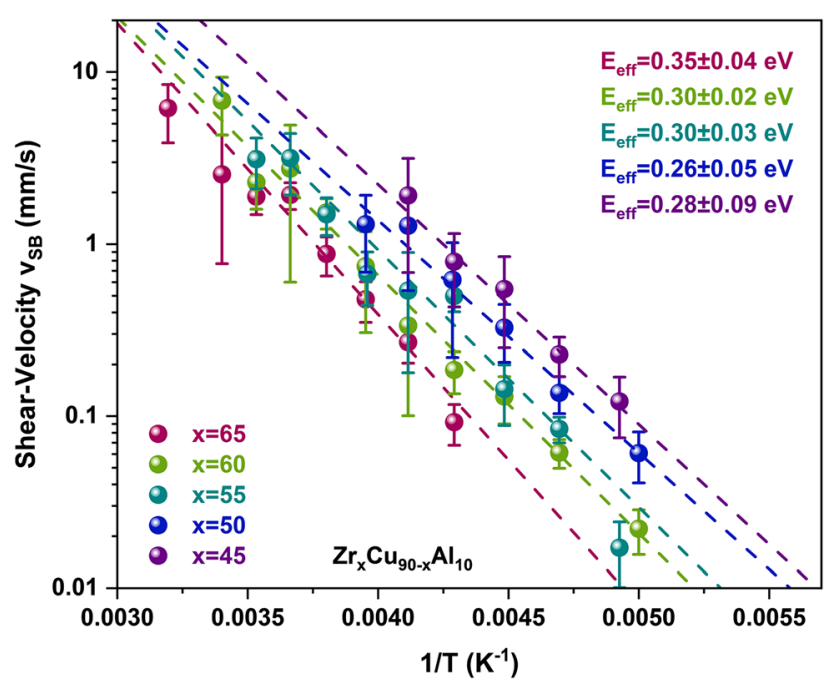

Fig. 3- Shear-band velocity as a function of inverse temperature for the $\mathrm{Zr}_{x} \mathrm{Cu}_{90-x} \mathrm{Al}_{10}$ system, revealing chemistry dependent effective barrier energies (Color figure online). 
observed at ca. $0.00425 \mathrm{~K}^{-1}\left(-40{ }^{\circ} \mathrm{C}\right)$. Consequently, the alloy with 65 at. pet $\mathrm{Zr}$ has the slowest and most stable shear response at this temperature, which translates to the highest plastic strain at failure at room temperature. ${ }^{[38]}$ It is noted that Reference 48 covers a larger temperature window than shown in Figure 3, which leads to slightly different $E_{\text {eff-values after fitting }}$ to an Arrhenius model. Whilst the data in Figure 3 is only an observation for one alloy system, one may naturally ask if this is a finding of potential general validity.

To expand the findings contained in Figure 3 to a broader class of alloys is an experimental task of considerable effort, which we will report on in a forthcoming manuscript. ${ }^{[49]}$ However, a simple literature study will reveal a series of effective barrier energies that are listed in Table I. This summary of values ranges from 0.26 to $0.48 \mathrm{eV}$, and I find it worthwhile to think about the fundamental structural parameters that may govern the value of $E_{\text {eff. }}$ This is of particular interest, since we hypothesize based on the data in Figure 3 that the shear stability of a metallic glass will largely depend on $E_{\text {eff }}$, and that the final strain at failure at room temperature may grow (or find a maximum) with increasing $E_{\text {eff }}$.

The typically constructed scaling of characteristic BMG-properties, such as a mechanical property with $T_{g}$, are insufficient to rationalized the trend of $E_{\text {eff }}$ in Figure 3 and Table I, which was discussed in Reference 51. This directs focus to the chemical and topological details of the alloy structure and one can begin with considering the nearest neighbor shell and the resistance it may have against disruption (Figure 4). Hypothesizing that the $E_{\text {eff }}$ is a measure of the barrier for diffusive jumps that are species dependent (here $\mathrm{Cu}, \mathrm{Zr}, \mathrm{Al}$ ), it is as a first step instructive to identify the fastest diffusing element. Based on the atomic size, the most ideal metallic bonding character, and selected atomistic studies, ${ }^{[52,53]}$ it is most reasonable that $\mathrm{Cu}$ is the fasted diffusor. With this assumption the second step consists of interrogating a composition with respect to how easy changes in local bonding environments are. This is determined by the binding energies between species and also the size of the central atom, since a large atom has a

Table I. Effective Barrier Energies Obtained from Shear-Band Dynamics Experiments on Various MG-Alloys

\begin{tabular}{lcc}
\hline Alloy & $E_{\text {eff }}$ & Referneces \\
\hline $\mathrm{Co}_{78} \mathrm{~B}_{12} \mathrm{Si}_{10}$ & $0.48 \pm 0.05$ & 2 \\
$\mathrm{Ni}_{70} \mathrm{Fe}_{8} \mathrm{~B}_{12} \mathrm{Si}_{10}$ & $0.46 \pm 0.05$ & 2 \\
$\mathrm{Zr}_{65} \mathrm{Cu}_{15} \mathrm{Ni}_{10} \mathrm{Al}_{10}$ & $0.37 \pm 0.04$ & 50 \\
$\mathrm{Zr}_{65} \mathrm{Cu}_{25} \mathrm{Al}_{10}$ & $0.35 \pm 0.03$ & 48 \\
$\mathrm{Zr}_{52.5} \mathrm{Cu}_{17.9} \mathrm{Ni}_{14.6} \mathrm{Al}_{10} \mathrm{Ti}_{5}$ & $0.32 \pm 0.00$ & 21 \\
$\mathrm{Zr}_{60} \mathrm{Cu}_{30} \mathrm{Al}_{10}$ & $0.32 \pm 0.03$ & 48 \\
$\mathrm{Zr}_{55} \mathrm{Cu}_{35} \mathrm{Al}_{10}$ & $0.28 \pm 0.02$ & 48 \\
$\mathrm{Zr}_{50} \mathrm{Cu}_{40} \mathrm{Al}_{10}$ & $0.26 \pm 0.05$ & 48 \\
$\mathrm{Zr}_{45} \mathrm{Cu}_{45} \mathrm{Al}_{10}$ & $0.28 \pm 0.09$ & 48 \\
\hline
\end{tabular}

The original Ref. [50] for $\mathrm{Zr}_{65} \mathrm{Cu}_{15} \mathrm{Ni}_{10} \mathrm{Al}_{10}$ reported a higher value and was re-evaluated for a meaningful comparison across all alloys, as outlined in Ref. [51]. larger coordination number. Therefore, $\mathrm{Cu}$ is surrounded by fewer atoms in Figure 4 than $\mathrm{Zr}$. Chemistry-specific coordination numbers can be estimated via the efficient-cluster-packing model, ${ }^{[54,55]}$ and this analysis reveals that the compositional effect on the bonding around $\mathrm{Cu}$ to its nearest neighbors changes according to the change of nominal composition in the $\mathrm{Zr}-\mathrm{Cu}-\mathrm{Al}$ system. With increasing $\mathrm{Zr}$-content, this leads to a shifting bond character from a $\mathrm{Cu}-\mathrm{Cu}$-like to a more $\mathrm{Cu}-\mathrm{Zr}$-like environment.

Using the mixing enthalpies and the heats of formation, one finds that $\mathrm{Cu}-\mathrm{Zr}$ bonds are stronger than $\mathrm{Cu}-\mathrm{Cu}$ bonds, which allows to conclude that $\mathrm{Cu}$ will experience a shallower migration barrier in the $\mathrm{Zr}$-lean alloy. Even though the assessed shear-band dynamics is a competition between athermal shear-disordering and thermally-activated relaxation, $E_{\text {eff }}$ naturally characterizes the latter. It thus follows that Zr-rich alloys in $\mathrm{Zr}_{x} \mathrm{Cu}_{90-x} \mathrm{Al}_{10}$ are expected to relax faster than $\mathrm{Zr}$-lean compositions, thereby increasing the resistance to continued shear. This is in good agreement with the data contained in Figure 3, providing a first atomistic pathway to understand and connect $E_{\text {eff }}$ to structural details of a metallic glass.

The mathematical details of the above structural analysis and its link to $E_{\text {eff }}$ have been described by Thurnheer et al., ${ }^{[38]}$ but suffer currently from two shortcomings: (1) It does not take into account the dilated structure in the shear-band core, and (2) it further does not include any bias of stress. Comparing between different alloys, the former can be ignored if one assumes a homogenous volume expansion and the absence of chemical shear-band segregation. ${ }^{[56]}$ The latter can be considered by the most basic relation $Q=H-\Omega \tau$, where $H$ is the zero-stress barrier, $\Omega$ the activation volume, $\tau$ the shear stress, and $\Omega$ a barrier energy. The three unknowns, $H, \Omega$ and $\tau$ can be determined independently from either experiments, or the efficient-packing-cluster model, which allows a refined structural model including a stress-bias that is currently underway.

As part of the experimentalists view, we add that the yield stress is connected to the shear modulus $G,{ }^{[57]}$ that itself obeys the theory of elastic modulus inheritance, ${ }^{[58]}$

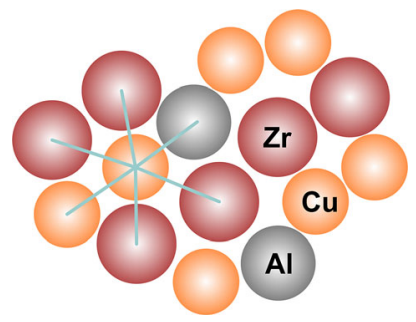

Fig. 4-A schematic of a possible bonding environment of $\mathrm{Cu}$ in a ternary BMG. We here pursue the idea that the nearest neighbor bonding environment determines the effective barrier energy obtained from data as shown in Fig. 3. The smaller $\mathrm{Cu}$ atom has a lower average coordination number than $\mathrm{Zr}$, and based on mixing enthalpies and the heats of formation, one finds that $\mathrm{Cu}-\mathrm{Zr}$ bonds are stronger than $\mathrm{Cu}-\mathrm{Cu}$ bonds. An increasing $\mathrm{Zr}$-content is therefore expected to strengthen the local environment and hence result in a larger $E_{\text {eff }}$ (Color figure online). 
which in the ternary case means that Zr-rich alloys have a lower shear modulus. One therefore finds lower $G$ values linked to a more sluggish shear-dynamics. Since low shear moduli often scale with the ductility of metallic glasses, ${ }^{[59,60]}$ the here sketched thought-process may suggest that an atomic-scale understanding for ductility and the design of ductile metallic glasses is within reach, where $E_{\text {eff }}$ plays a central role. Based on almost 20 different compositions, we will demonstrate this opportunity in detail in the future. ${ }^{[49]}$

\section{Length Scales of the Underlying Shear Defect}

Serrated flow in metallic glasses has for a long time revolved around the mechanics and the detailed appearance of the stress-strain signature, whereas the underlying structure, and in particular the mediating shear defect, received experimentally less attention. One obvious reason for this is the difficulty in revealing and characterizing a nano-scale sheared region in a disordered material. In the context of structural heterogeneities of metallic glasses, this has been summarized in detail in a recent review article, ${ }^{[61]}$ and we will here only highlight a few aspects regarding the fluctuations of both length scales and structural measures of shear bands.

At the smallest scale of the shear-band core, detailed scanning-transmission electron microscopy (STEM) work has revealed that the simple measure of the shear-band thickness $t_{w}$ can vary more than 40 pct over a distance of $600 \mathrm{~nm}$, and become a tenfold of the commonly cited shear-band thickness of 10 or $20 \mathrm{~nm} .^{[62]}$ One may argue that deviations away from 10 to $20 \mathrm{~nm}$ can be caused by projection errors during STEM imaging, but tilting the TEM sample allows the determination of an edge-on view with the electron beam, as discussed in Reference 63. This variation of the width of the shear-band core is schematically depicted in Figure 5, to which we will add two additional length scales of substantially larger values.

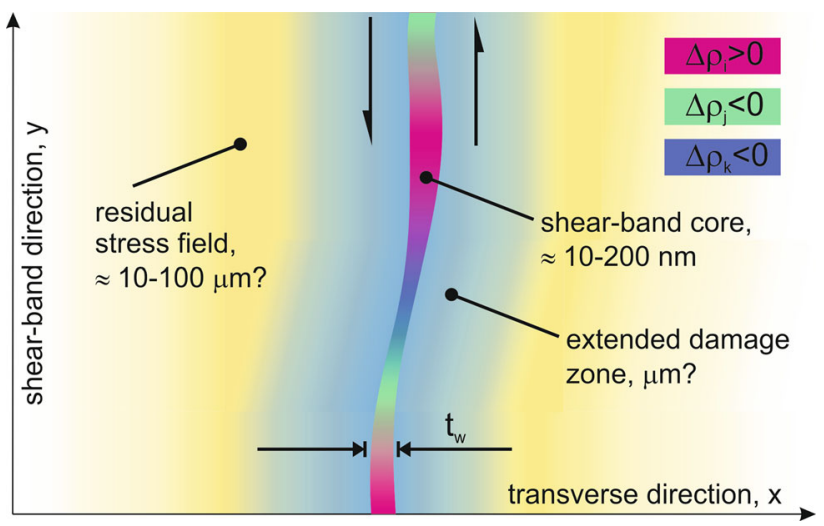

Fig. 5-Schematic indicating a shear-band core surrounded by some yet not well quantified extended damage zone, and a long-range residual stress field. The shear-band thickness $t_{w}$ and the volumetric change of the shear-band material $\Delta \rho$ vary along the shear-band direction $y$. Note that this is a simplified cross-sectional view of a planar defect (Color figure online).
With the nanoindentation work by Pan et al. ${ }^{[64]}$ and Maass et al. ${ }^{[65]}$ it became clear that there is some long-range signature around the nano-scopic shearband core, extending over tens to hundred micrometers. These initial reports had different interpretations, but captured the same feature. Our own conclusion was that the long-range signature measured via nanoindentation hardness maps originated from position-dependent residual stresses, of which the in-plane component is known to cause changes in the measured hardness. ${ }^{[66]}$ These residual stresses were proposed to emerge when shear occurs over a non-planar shear-band plane, ${ }^{[27]}$ locally giving rise to either tensile or compressive stress states. That means, no structural change was ascribed to the long-range softening (or hardening). A long-range strain field around a shear band was also reported via $\mathrm{X}$-ray strain mapping ${ }^{[67]}$ and magnetic force microscopy. ${ }^{[68]} \mathrm{X}$-ray correlation spectroscopy suggests that these long-range stress (or strain) fields can significantly enhance the local relaxation response of the matrix material around the shear band itself. ${ }^{[6]}$ A critical aspect in the quantification of these still unsatisfactorily understood and characterized long-range signatures is that they depend on location, meaning that they may not even be observed in a single experiment. Similar to the later discussed shear-band structure, strongly position-dependent properties increase the complexity of the shear defect. A sensible contribution to the question if the long-range signatures are caused by only residual internal stresses or also structural changes was recently provided via fluctuation microscopy. ${ }^{[70]}$ These measurement indicated a micron-wide damage zone around the shear-band core that indeed revealed structural changes. We emphasize that this is a distinctly different observation than the signature of a long-range residual stress field.

These developments do not only motivate the distinction between a nanometer shear-band core, a surrounding micrometer structural damage zone, and an even larger zone of residual stresses, as schematically summarized in Figure 5, but also that the length-scales of these are position-dependent properties. In particular, the latter insight makes the experimental characterization of this shear defect very challenging, since results will depend on where they are measured along the shear-path. As we will see in the following section, the emerging locality of the shear-band is also a dominant factor in the structural change.

\section{Local Shear-Band Structure}

Focusing on the underlying shear defect instead of the stress-strain signature naturally leads to interrogating the structure of a formed shear band. One experimentally unexplored aspect of shear-band structure is the question if any possible damage accumulation occurs during shear. In other words, is the structure of the shear band evidencing any systematic change with locally admitted shear strain $\gamma$ ? Our work on shear-band dynamics repeatedly revealed indications for that this may be the case, as for example seen in Figure. 3 of Reference 20 where $v_{\mathrm{SB}}$ increases with strain towards 
failure. Selected atomistic simulations have interrogated regions of strain localization as a function of local shear strain, ${ }^{[71,72]}$ but to our knowledge, no experimental data set has been reported. One of the few methods that allows quantitative tracking of shear-band structure is high-angle annular dark-field (HAADF) STEM that can return changes in density $(\Delta \rho)$ between the shear-band core and the surrounding matrix material. ${ }^{[73]}$ This approach has revealed both significant reductions in density with amounts exceeding $\sim 10$ pct, ${ }^{[62,73]}$ but also regions of increased density $(\sim 6 \mathrm{pct}) \cdot{ }^{[74]}$ Traced along the shear-band, $\Delta \rho$ is like the shear-band thickness a locally varying property. ${ }^{[62]}$ Using HAADF-STEM on shear-bands that have admitted different amounts of shear strain, we pursued a statistical assessment of both local $\Delta \rho$ and $t_{w}$ values in a $\mathrm{Zr}$-based BMG $\left(\mathrm{Zr}_{52.5} \mathrm{Cu}_{17.9-}\right.$ $\mathrm{Ni}_{14.6} \mathrm{Al}_{10} \mathrm{Ti}_{5}$, Vit105). One key aspect of this effort was to sample a large range of $\gamma$, which initially was attempted by varying the amount of axial engineering strain of compression samples. ${ }^{[75]}$ In cases where only one single shear band accumulated all plastic strain, it is straight forward to calculate a local $\gamma$ of the shear band using the locally measured shear-band thickness. Figure 6 shows this data set as a cluster at shear strains larger than 1000. This approach involves the complication of finding a shear band in a cross-sectional cut of a bulk sample, the procedure of which is outlined in Reference 62.

A different approach using 3-point bending was subsequently pursued to assess smaller shear-strains, which has the advantage that a TEM lamella can be extracted directly at the surface step. That means, the magnitude of the shear-band surface-steps resulting from plastic bending can be determined and sampled in order to probe different $\gamma$. A TEM sample lift-out is done including the surface step, from which a shearband segment can be traced inwards into the sample. In this way, $\gamma$-values below 100 were probed. The

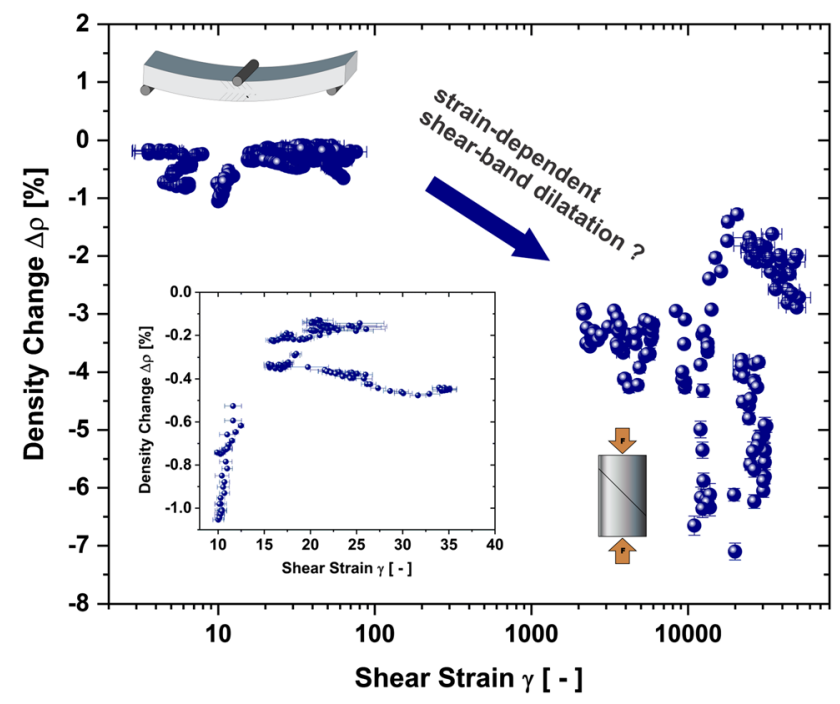

Fig. 6-Density change as a function of shear strain for TEM data extracted from both the 3-point bending sample and compression samples. The inset highlights the data for the bending sample (Color figure online) corresponding data is displayed in the upper left corner in Figure 6. In addition to the much smaller shear-strain values, it is also clear that the associated local density changes are much smaller, mostly yielding values that are between the resolution limit and $\sim 1$ pct density decrease. It is finally interesting to note that no compaction (positive $\Delta \rho$ ) was measured as part of this data set, but has been seen in our work on a ternary Zr-based alloy. ${ }^{[63]}$

Each data set alone in Figure 6 does not reveal any particular trend, and also data sets from individual TEM foils do not allow any systematic conclusion. A selection of three different shear-bands is highlighted in the inset in Figure 6, demonstrating no change of $\Delta \rho$ with increasing $\gamma$, no change of $\gamma$ with decreasing $\Delta \rho$, or a mildly decreasing $\Delta \rho$ with increasing $\gamma$. However, the two strongly scattered data sets together hint towards some overall trend. What structural mechanisms may drive this scatter remains experimentally inaccessible, and clearly more efforts are needed to investigate possible dependencies between structure and shear strain. However, a direct conclusion from this data is that single measurement may reveal any result, and that generalized conclusions based on small data sets cannot be made. The large scatter in Figure 6 is therefore another manifestation of the locality of shear deformation that was discussed in the previous section.

Whilst the here presented data is in qualitative agreement with recent computer simulations that highlight the position-dependent complexity of strain-localization in $\mathrm{BMGs},{ }^{[76-78]}$ we are far away from understanding the local mechanisms that cause these fluctuations across much larger length-scales than of short- and medium-range structural order. To overcome this hurdle with experiments does currently not seem feasible, and substantial efforts/developments in atomistic simulations are needed in view of the length scales, time scales, and the prominence of thermal activation in shear banding. In fact, one has to conclude that current atomistic simulations never capture the same damage mechanism as characterized with experiments. With our continued effort in probing true thermally-activated structural activity in metallic glasses via micro-second time-scale molecular dynamics simulations, ${ }^{[79-81]}$ we are currently exploring sufficiently low strain rates that can produce a serrated flow response that is not simply a manifestation of driven and athermal plasticity.

\section{CONCLUDING REMARKS AND OUTLOOK}

This manuscript discusses four research directions that go beyond the stress-strain signature of serrated flow in BMGs.

We began with the statistical characteristics of plastic fluctuations in serrated flow. The current status of this approach suggests some scale-free response of ductile BMGs, but also reveals as of now not well understood structural influences on the serration statistics. This itself warrants further studies, as an apparent contradiction between microstructurally insensitive theoretical statistical models and structural effects on the statistics 
emerge. It remains also unclear what alloy-specific microstructural mechanisms dictate long-range interactions and correlated plastic activity that scale-invariance entails.

As a second direction, we articulated the possibility of using the effective barrier energy derived from temperature dependent shear-band velocities as a descriptor for shear stability. If we are successful with proving the validity of this approach, a model would be within reach that connects experimentally derived quantities with the atomistic details of the alloy. One may further speculate if such a model could guide the design of malleable BMG compositions.

The third and fourth research directions are related, as they address length scales and the structure of shear bands that underlie serrated flow. Here it becomes clear that a shear band is much more complex than a simple plane with a finite thickness and uniform properties. All experimentally so far revealed properties are depending on position, and the origins of the various signatures beyond the nano-scale shear-band core are yet to be unraveled. We end with posing the question if structural damage accumulates with strain inside a shear band. Given evidence of structural changes outside the shear-band core, this question may be also be considered at the larger length-scales of an extended damage zone.

We would argue that serrated flow and the dynamics of shear bands is a very well understood topic. However, many fundamental questions relating to the strain-localization process remain elusive or are essentially unexplored. Metallic glasses fall, in this regard, well behind their crystalline counterparts, for which textbooks have been filled with detailed defect mechanisms. If the international research community wants to live up to the originally articulated vision of developing ductile glassy alloys, continued efforts are needed to fill this gap. We hope that this contribution can spark new efforts in understanding plastic flow of metallic glasses.

\section{ACKNOWLEDGMENTS}

This research was carried out in part in the Frederick Seitz Materials Research Laboratory Central Research Facilities at UIUC. RM gratefully acknowledges start-up funds provided by the Department of Materials Science and Engineering at UIUC. RM acknowledges the excellent co-workers that have contributed to the here discussed topic, including J.F. Löffler, D. Klaumünzer, P. Thurnheer, K. Laws, P.M. Derlet, C. Liu, S. Küchemann, A. Das, C. Volkert, V. Roddatis, K. Samwer, and many more.

\section{FUNDING}

Open Access funding provided by Projekt DEAL.

\section{OPEN ACCESS}

This article is licensed under a Creative Commons Attribution 4.0 International License, which permits use, sharing, adaptation, distribution and reproduction in any medium or format, as long as you give appropriate credit to the original author(s) and the source, provide a link to the Creative Commons licence, and indicate if changes were made. The images or other third party material in this article are included in the article's Creative Commons licence, unless indicated otherwise in a credit line to the material. If material is not included in the article's Creative Commons licence and your intended use is not permitted by statutory regulation or exceeds the permitted use, you will need to obtain permission directly from the copyright holder. To view a copy of this licence, visit http://creat ivecommons.org/licenses/by/4.0/.

\section{REFERENCES}

1. H. Kimura and T. Masumoto: Philos. Mag. A, 1981, vol. 44, pp. 1005-20.

2. H. Kimura and T. Masumoto: Philos. Mag. A, 1981, vol. 44, pp. 1021-30.

3. H. Kimura and T. Masumoto: Acta Metall., 1983, vol. 31, pp. $231-40$.

4. B.J. Brindley and J.T. Barnby: Acta Metall., 1966, vol. 14, pp. $1765-80$.

5. P.G. McCormic: Acta Metall., 1971, vol. 19, pp. 463-71.

6. G. Sparks, Y. Cui, G. Po, Q. Rizzardi, J. Marian, and R. Maass: Phys. Rev. Mater., 2019, vol. 3, art. no. 080601

7. C.A. Schuh, A.S. Argon, T.G. Nieh, and J. Wadsworth: Philos. Mag., 2003, vol. 83, pp. 2585-97.

8. C.A. Schuh, A.C. Lund, and T.G. Nieh: Acta Mater., 2004, vol. 52, pp. 5879-91.

9. F. Shimizu, S. Ogata, and J. Li: Acta Mater., 2006, vol. 54, pp. 4293-98.

10. W.H. Jiang, G.J. Fan, F.X. Liu, G.Y. Wang, H. Choo, and P.K. Liaw: Int. J. Plast., 2008, vol. 24, pp. 1-16.

11. W.J. Wright, M.W. Samale, T.C. Hufnagel, M.M. LeBlanc, and N. Florando: Acta Mater., 2009, vol. 57, pp. 4639-48.

12. W.H. Jiang, G.J. Fan, F.X. Liu, G.Y. Wang, H. Choo, and P.K. Liaw: J. Mater. Res., 2006, vol. 21, pp. 2164-67.

13. H.M. Chen, J.C. Huang, S.X. Song, T.G. Nieh, and J.S.C. Jang: Appl. Phys. Lett., 2009, vol. 94, art. no. 141914.

14. H. Neuhäuser: Scr. Metall., 1978, vol. 12, pp. 471-74.

15. W.J. Wright, R.B. Schwarz, and W.D. Nix: Mater. Sci. Eng. A, 2001, vol. 319, pp. 229-32.

16. B. Yang, M.L. Morrison, P.K. Liaw, R.A. Buchanan, G.Y. Wang, C.T. Liu, and M. Denda: Appl. Phys. Lett., 2005, vol. 86, art. no. 141904.

17. W.H. Jiang, H.H. Liao, F.X. Liu, H. Choo, and P.K. Liaw: Metall. Mater. Trans. A, 2008, vol. 39A, pp. 1822-30.

18. Y. Zhang, N.A. Stelmashenko, Z.H. Barber, W.H. Wang, J.J. Lewandowski, and A.L. Greer: J. Mater. Res., 2007, vol. 22, pp. 419-27.

19. K. Georgarakis, M. Aljerf, Y. Li, A. LeMoulec, F. Charlot, A.R. Yavari, K. Chornokhvostenko, E. Tabachnikova, G.A. Evangelakis, D.B. Miracle, A.L. Greer, and T. Zhang: Appl. Phys. Lett., 2008, vol. 93, art. no. 031907.

20. D. Klaumünzer, R. Maass, F.H. DallaTorre, and J.F. Löffler: Appl. Phys. Lett., 2010, vol. 96, p. 61901.

21. R. Maass, D. Klaumünzer, and J.F. Löffler: Acta Mater., 2011, vol. 59, pp. 3205-13.

22. R. Maass and J.F. Löffler: Adv. Func. Mater., 2015, vol. 25, pp. 2353-68. 
23. P. Thurnheer, F. Haag, and J.F. Löffler: Acta Mater., 2016, vol. 115 , pp. $468-74$.

24. A. Das, P. Kagebein, S. Kuechemann, and R. Maass: Appl. Phys. Lett., 2018, vol. 112, art. no. 261905

25. R. Maass, D. Klaumünzer, G. Villard, P.M. Derlet, and J.F. Löffler: Appl. Phys. Lett., 2012, vol. 100, art. no. 071904.

26. R.T. Qu, S.G. Wang, X.D. Wang, Z.Q. Liu, and Z.F. Zhang: Scripta Mater., 2017, vol. 133, pp. 24-28.

27. C. Liu, A. Das, W. Wang, S. Küchemann, P. Kenesei, and R. Maass: Scripta Mater., 2019, vol. 170, pp. 29-33.

28. J. Brechtl, S.Y. Chen, X. Xie, Y. Ren, J.W. Qiao, P.K. Liaw, and S.J. Zinkle: Int. J. Plast, 2019, vol. 115, pp. 71-92.

29. S. Chen, X. Xie, B. Chen, J. Qiao, Y. Zhang, Y. Ren, K.A. Dahmen, and P.K. Liaw: JOM, 2015, vol. 67, pp. 2314-20.

30. Q. Rizzardi, G. Sparks, and R. Maass: JOM, 2018, vol. 70 , pp. 1088-93.

31. J.P. Sethna, M.K. Bierbaum, K.A. Dahmen, C.P. Goodrich, J.R. Greer, L.X. Hayden, J.P. Kent-Dobias, E.D. Lee, D.B. Liarte, X. Ni, K.N. Quinn, A. Raju, D. ZebRocklin, A. Shekhawat, and S. Zapperi: Annu. Rev. Mater. Res., 2017, vol. 47 , pp. $217-46$.

32. R. Maass and P.M. Derlet: Acta Mater., 2018, vol. 143, pp. $338-63$.

33. M. Zaiser, J. Schwerdtfeger, A.S. Schneider, C.P. Frick, B.G. Clark, P.A. Gruber, and E. Arzt: Philos. Mag., 2008, vol. 88, pp. 3861-74.

34. D.V. Denisov, K.A. Lorincz, J.T. Uhl, K.A. Dahmen, and P. Schall: Nat. Commun., 2016, vol. 7, art. no. 10641.

35. B.A. Sun, H.B. Yu, W. Jiao, H.Y. Bai, D.Q. Zhao, and W.H. Wang: Phys. Rev. Lett., 2010, vol. 105, art. no. 189904.

36. J. Antonaglia, W.J. Wright, X. Gu, R.R. Byer, T.C. Hufnagel, M. LeBlanc, J.T. Uhl, and K.A. Dahmen: Phys. Rev. Lett., 2014 vol. 112 , art. no. 155501 .

37. G. Wang, K.C. Chan, L. Xia, P. Yu, J. Shen, and W.H. Wang: Acta Mater., 2009, vol. 57, pp. 6146-55.

38. P. Thurnheer, R. Maass, K.J. Laws, S. Pogatscher, and J.F. Löffler: Acta Mater., 2015, vol. 96, pp. 428-36.

39. J. Antonaglia, X. Xie, G. Schwarz, M. Wraith, J. Qiao, Y. Zhang, P.K. Liaw, J.T. Uhl, and K.A. Dahmen: Sci. Rep., 2014, vol. 4, art. no. 4382.

40. W.F. Wu, Y. Li, and C.A. Schuh: Philos. Mag., 2008, vol. 88, pp. 71-89.

41. K. Mondal and K. Hono: Mater. Trans., 2009, vol. 50, pp. 152-57.

42. Z. Wang, J.W. Qiao, G. Wang, K.A. Dahmen, P.K. Liaw, Z.H. Wang, B.C. Wang, and B.S. Xu: Mater. Sci. Eng., A, 2015, vol. 639 , pp. $663-70$.

43. L. Zhang, R.L. Narayan, H.M. Fu, U. Ramamurty, W.R. Li, Y.D. Li, and H.F. Zhang: Acta Mater., 2019, vol. 168, pp. 24-36.

44. W.J. Wright, A.A. Long, X. Gu, X. Liu, T.C. Hufnagel, and K.A. Dahmen: J. Appl. Phys., 2018, vol. 124, art. no. 185101.

45. P.S. Singh, R.L. Narayan, I. Sen, D.C. Hofmann, and U. Ramamurty: Mater. Sci. Eng. A, 2012, vol. 534, pp. 476-84.

46. G. Sparks and R. Maass: Phys. Rev. Mater., 2018, vol. 2, art. no. 120601.

47. G. Sparks and R. Maass: Eur. Phys. J. B, 2019, vol. 92, art. no. 15.

48. P. Thurnheer, R. Maass, S. Pogatscher, and J.F. Löffler: Appl. Phys. Lett., 2014, vol. 104, art. no. 101910.

49. P. Thurnheer, R. Maass, K. Laws, J. F. Loeffler: unpublished data, 2017.

50. B.A. Sun, S. Pauly, J. Hu, W.H. Wang, U. Kuhn, and J. Eckert: Phys. Rev. Lett., 2013, vol. 110, art. no. 225501.
51. P. Thurnheer: ETH Zurich, 2016, PhD Thesis.

52. J. Ding, Y. Cheng, and E. Ma: Acta Mater., 2014, vol. 69, pp. 343-54.

53. J. Ding, Y. Cheng, and E. Ma: Acta Mater., 2013, vol. 61, pp. $4474-80$

54. D.B. Miracle: Acta Mater., 2006, vol. 54, pp. 4317-36.

55. K.J. Laws, D.B. Miracle, and M. Ferry: Nat. Commun., 2015, vol. 6 , art. no. 8123 .

56. C. Liu, Z. Cai, X. Xia, V. Roddatis, R. Yuan, J.M. Zuo, and R. Maass: Scripta Mater., 2019, vol. 169, pp. 23-27.

57. W.L. Johnson and K. Samwer: Phys. Rev. Lett., 2005, vol. 95, art. no. 195501

58. D. Ma, A.D. Stoica, X.L. Wang, Z.P. Lu, B. Clausen, and D.W. Brown: Phys. Rev. Lett., 2012, vol. 108, art. no. 085501.

59. J.J. Lewandowski, W.H. Wang, and A.L. Greer: Philos. Mag. Lett., 2005, vol. 85, pp. 77-87.

60. S.J. Poon, A. Zhu, and G.J. Shiflet: Appl. Phys. Lett., 2008, vol. 92, art. no. 261902.

61. C. Liu and R. Maass: Adv. Func. Mater., 2018, vol. 28, art. no. 1800388.

62. C. Liu, V. Roddatis, P. Kenesei, and R. Maass: Acta Mater., 2017, vol. 140 , pp. $206-16$

63. C. Liu: UIUC, 2020, PhD Thesis.

64. J. Pan, Q. Chen, L. Liu, and Y. Li: Acta Mater., 2011, vol. 59, pp. 5146-58.

65. R. Maass, P. Birckigt, C. Borchers, K. Samwer, and C.A. Volkert: Acta Mater., 2015, vol. 98, pp. 94-102.

66. T.Y. Tsui, W.C. Oliver, and G.M. Pharr: J. Mater. Res., 1996, vol. 11 , pp. $752-59$.

67. H. Shahabi, S. Scudino, I. Kaban, M. Stoica, B. Escher, S. Menzel, G.B.M. Vaughan, U. Kühn, and J. Eckert: Acta Mater., 2016, vol. 111, pp. 187-93.

68. L.Q. Shen, P. Luo, Y.C. Hu, H.Y. Bai, Y.H. Sun, B.A. Sun, Y.H. Liu, and W.H. Wang: Nat. Commun., 2018, vol. 9, p. 4414.

69. S. Kuechemann, C. Liu, E. Dufresne, J. Shin, and R. Maass: Phys. Rev. B, 2018, vol. 97, art. no. 014204 .

70. F.A. Davani, S. Hilke, H. Rösner, D. Geissler, A. Gebert, and G. Wilde: J. Alloy. Compd., 2020, vol. 837, art. no. 155494

71. Y. Shi, M.B. Katz, H. Li, and M.L. Falk: Phys. Rev. Lett., 2007, vol. 98 , art. no. 185505.

72. D.D. Alix-Williams and M.L. Falk: Phys. Rev. E, 2018, vol. 98, art. no. 053002 .

73. H. Rösner, M. Peterlechner, C. Kuebel, V. Schmidt, and G. Wilde: Ultramicroscopy, 2014, vol. 142, pp. 1-9.

74. V. Schmidt, H. Roesner, M. Peterlechner, G. Wilde, and P.M. Voyles: Phys. Rev. Lett., 2015, vol. 115, art. no. 035501.

75. C. Liu, Y. Ikeda, and R. Maass: Scr. Mater, 2021, vol. 190, pp. 75-79.

76. M. Hassani, A.E. Lagogianni, and F. Varnik: Phys. Rev. Lett., 2019, vol. 123, art. no. 195502.

77. D. Sopu, A. Stukowski, M. Stoica, and S. Scudino: Phys. Rev. Lett., 2017, vol. 119, art. no. 195503.

78. P.M. Derlet and R. Maass: Acta Mater., 2018, vol. 143, pp. 205-13.

79. P.M. Derlet and R. Maass: J. Mater. Res., 2017, vol. 32, pp. $2668-79$.

80. P.M. Derlet and R. Maass: J. Alloy. Compd., 2020, vol. 821, art. no. 153209

81. P.M. Derlet and R. Maass: Materialia, 2018, vol. 3, pp. 97-106.

Publisher's Note Springer Nature remains neutral with regard to jurisdictional claims in published maps and institutional affiliations. 